\title{
Monitoring of a skin temperature in vicinity of a surgical wound
}

\author{
David Tolar \\ New Technologies - Research Centre \\ University of West Bohemia \\ Plzen, Czech Republic \\ dtolar@ntc.zcu.cz
}

\author{
Milan Stork \\ Dept. Applied Electronics and Telecommunications \\ University of West Bohemia \\ Plzen, Czech Republic \\ stork@kae.zcu.cz
}

\begin{abstract}
This article describes experimental measurements of a skin temperature in vicinity of a surgical wound. Measurements were carried out in collaboration with the orthopedic department of the University Hospital Olomouc, Czech Republic. A system used for sensing, transmission and visualization of physiological parameters was developed at the University of West Bohemia in Pilsen. A main goal of measurements was to evaluate technical parameters of the system, and also non-technical aspects associated with the use of the system. Technical parameters that was verified was a reliability of the system as a whole. This covers a temperature and motion sensing, a wireless transfer of measured data from sensors through wireless routers to the data server and data visualization in web application. To asses non-technical aspects of the system use, a sociological survey was carried out. Patients were asked to answer several questions related to the use of the system and temperature sensors. Conducted series of measurements and the survey showed that the vast majority of patients are accepting new technology positively. Measurements also verified the usability of the temperature sensor for local skin temperature measurement. Furthermore measurements revealed some issues associated with use of the sensor for monitoring of human body temperature and set a new target for further research and development.
\end{abstract}

Keywords - sensor; temperature; skin; skin temprature; sensor network; health care

\section{INTRODUCTION}

Presented measurements were part of a project focused on implementation of new technologies leading to increase quality of life with lowering socioeconomic costs. Based on an existing experience from previous clinical measurements a methodic was proposed, which allowed a long term measurements and a data acquisition using the system for continuous monitoring and visualization of skin temperature and motion activity. Measurements were carried out in cooperation with patients after knee replacement surgical procedure. Different configurations of sensors placement around the knee surgical wound was tested. The final configuration of sensors, which was used during the measurements, is shown on Fig. 1 .

A reference measurement of body temperature was carried by medical staff several times a day and registered on a patient's measurement card. The body temperature measurement was carried using infrared thermometer Microlife NC120. The temperature was measured on patient's forehead. Measured temperatures were compared to temperatures acquired with temperature sensors.

An additional temperature sensor was used for a body temperature sensing. Medical stuff tried various locations of the sensor on patient's body and finally used an abdominal area for the reference measurement.

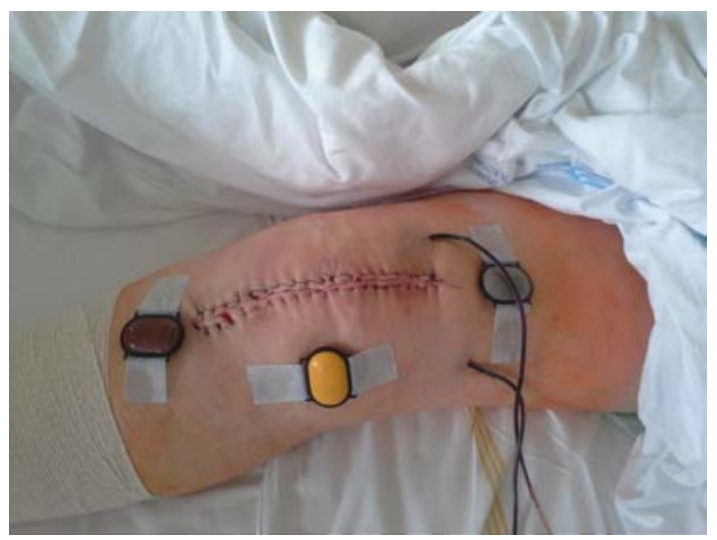

Figure 1 Placement of temperature sensors around the wound

\section{MEASUREMENT SYSTEM}

The measurement system is a wireless system, which uses miniature battery powered sensors to measure temperature and motion.

The system communicates in the $2.4 \mathrm{GHz}$ ISM frequency band and is compliant with IEEE Std 802.15.4TM - 2003, 2006 Wireless Medium Access Control (MAC) and Physical Layer (PHY) Specifications for Low-Rate Wireless Personal Area Networks (LR - WPANs) [3]. The communication rate is $500 \mathrm{kBit} / \mathrm{s}$.

The system [1] consists of following components: wireless sensors, wireless routers and a data server. Typical system setup is shown on Fig. 2.

The sensor is a low power wireless device [2], which allows temperature and motion activity measurement with user definable measurement period from one second to tens of minutes. Measurements were carried out with measurement period of 1 minute. A measured data from the sensor are passed to one of wireless routers, which is in communication range and transferred through the wireless network to 
the data server. The data are accessible through a web application accessible from any PC or mobile device connected to hospital local area network. Web application provides easy to understand access to patient's data through patient's card, which is shown on Fig. 3.

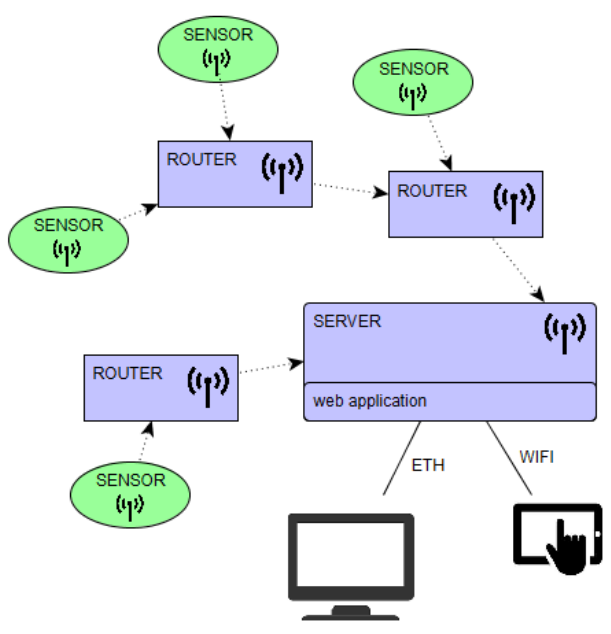

Figure 2 Typical measurement system setup

Patient's card signalize patient's condition through changing its color. If patient's temperature is above a predefined threshold, then the card highlights with red color. The system can also send an email, containing a warning information, in case that the temperature exceeds the threshold.

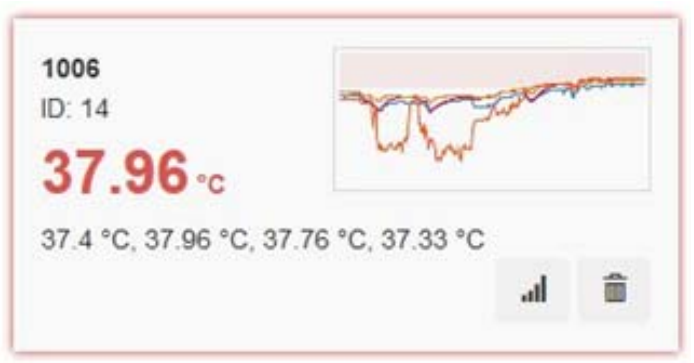

Figure 3 Patient card

The measured data can be evaluated in detail in an interactive chart shown on Fig. 4. The chart also allows adding of commented events inside the chart for better orientation in the data. Access to the web application interface is secured by username and password and the web application uses a https protocol to secure communication between the server and some client. The user interface of web application was designed in cooperation with medical staff to provide clean and simple view on measured data. The web application also signalizes special events like a delayed data reception and low battery of some sensor.

The temperature sensor used in measurements and its fixation on the skin is shown on figure Fig. 5. Dimensions of the sensor are $38 \times 25 \times 10 \mathrm{~mm}$. Fixation of the sensor on the skin was solved by using a standard medical tape. The sensor contains internal $3 \mathrm{~V}$ lithium battery with nominal capacity of 200 mAh. The battery lifetime depends on the measurement period. For 1 minute measurement period the lifetime of the battery is at least one year.
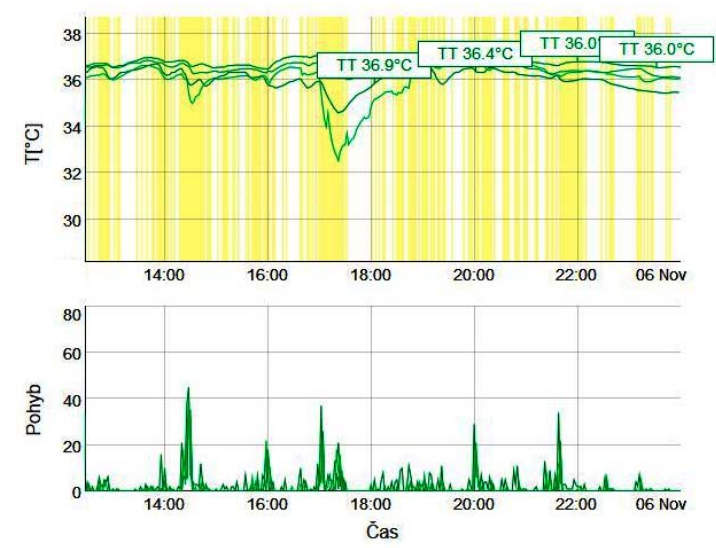

Figure 4 Interactive chart for data evaluation

The sensor also contains an 3-axis accelerometer for evaluation of patient's motion activity. Monitoring the motion activity has been found important, as patient's motion has some impact on the measured temperatures. Motion activity chart is shown in the patient's detail screen on Fig. 4. (lower part).

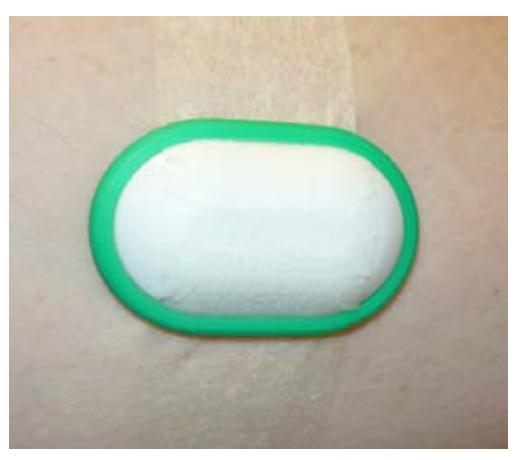

Figure 5 Temperature sensor fixated on skin

The accuracy of the skin temperature sensing is influenced by the quality and stability of the contact of sensor's temperature sensing surface with the skin and by the ambient temperature. The fixation by the medical tape is not sufficient in all conditions and causes changes in the heat transfer from the skin to the sensing surface of the sensor. These changes then cause temperature measurement errors.

The fixation by medical tape has been found as not sufficient and different fixation methods are investigated.

The influence of the ambient temperature is decreased by the construction of the sensor. The construction maximizes heat transfer between the sensing surface of the sensor and a temperature sensing element and on the other side minimizes heat transfer between sensor cover ant the temperature sensing element. Testing proved, that the construction alone is not sufficient to eliminate the ambient temperature influence. The temperature can vary up to $1{ }^{\circ} \mathrm{C}$ if the sensor is located freely in the air or covered by thermally isolating material like a bed blanket or a pillow. A method of compensation of this effect is now being investigated. 


\section{MEASUREMENT RESULTS}

Temperature measurements were carried out with three temperature sensors located near surgical wound as shown on Fig. 1. A fourth sensor was used to measure the body temperature. A placement of the sensor into the armpit, which is a clinically recognized location for sensing body temperature, was not feasible. The sensor cannot be well fixed at that location and a continuous and stable contact of the skin with the sensor's sensing surface can't be guaranteed. This is due to a shape of the armpit and a presence of hair in the armpit.

Some alternative locations was examined. Those were a location under the collarbone and in the abdominal area. Although not clinically recognized location, the abdominal area was chosen as the location of the sensor. The location is not clinically recognized for the body temperature measurement, nevertheless the temperature measured at this location can still be compared to the infrared thermometer readings from patient's forehead and in some cases detect a possible error in patient's body temperature.

The measured data was evaluated to find relation between the body temperature measurement taken by the infrared thermometer and temperatures from sensors. TABLE I shows a sample data for two patients. The column TD1 contains temperature difference between the sensor located in patient's abdominal area and the measurement manually taken by the infrared thermometer.

TABLE I. TEMPERATURE DIFFERENCES

\begin{tabular}{|c|c|c|c|c|c|}
\hline patient & TD1 $\left[{ }^{\circ} \mathbf{C}\right]$ & TD2 $\left[{ }^{\circ} \mathbf{C}\right]$ & patient & TD1 $\left[{ }^{\circ} \mathbf{C}\right]$ & TD2 $\left[{ }^{\circ} \mathbf{C}\right]$ \\
\hline 1014 & 0.28 & 0.34 & 1015 & -0.16 & 0.44 \\
\hline 1014 & -0.2 & 0.14 & 1015 & -1.04 & 0.5 \\
\hline 1014 & -1.44 & 0.39 & 1015 & -1.36 & 1.06 \\
\hline 1014 & -0.68 & -0.15 & 1015 & -2.96 & -0.75 \\
\hline 1014 & -1.05 & -0.26 & 1015 & -2.85 & -0.34 \\
\hline 1014 & -0.05 & 0.8 & 1015 & -1.82 & 0.9 \\
\hline 1014 & 0 & 0.77 & 1015 & -0.73 & -0.11 \\
\hline 1014 & -0.52 & 0.54 & 1015 & -0.2 & 1.06 \\
\hline 1015 & 0.14 & 0.6 & 1015 & 0.86 & 0.86 \\
\hline 1015 & 0.12 & 0.4 & 1015 & 0.36 & -0.04 \\
\hline 1015 & -0.27 & 0.08 & 1015 & -0.24 & 1.27 \\
\hline & & & 1015 & -11.58 & -0.67 \\
\hline
\end{tabular}

Differences has been divided into three intervals. First interval covers differences from $+0.4{ }^{\circ} \mathrm{C}$ to $-0.4{ }^{\circ} \mathrm{C}$. Values belonging to this interval are highlighted in green color and represents $26 \%$ of measurements. This interval is defined as a sum of measurement accuracy of the infrared thermometer and the sensor, which both were defined as $\pm 0.2^{\circ} \mathrm{C}$. Values within this interval correspond sufficiently with the infrared thermometer measurement.

Second interval covers differences lower than $-0.4^{\circ} \mathrm{C}$. Values belonging to this interval are highlighted in blue color and represents $67 \%$ of measurements. These differences are caused by errors, whose source can be inappropriate location of the sensor, faulty contact of sensor with skin, error caused by improper infrared temperature measurement or their mutual combination.

Third interval covers differences higher than $+0.4{ }^{\circ} \mathrm{C}$. Values belonging to this interval are highlighted in red color and represents $7 \%$ of measurements. These differences are probably caused by an inaccurate manual measurement of body temperature by the infrared thermometer. This assumption is based on a fact, that it's not likely, that a skin temperature in an abdominal area is higher than a body temperature. The possible cause like an external heat source present near the sensor was eliminated as the reason by medical stuff. The temperature taken by the sensor is always a bit lower than actual skin temperature.

An acceptable difference between temperatures from the infrared thermometer measurement and the sensor located in an abdominal area was found in only $26 \%$ of measurements. The result confirms that abdominal area is not suitable for body temperature measurement. Moreover the result also suggests, that in $7 \%$ of measurements, the infrared body temperature measurement can be inaccurate.

The column TD2 contains difference between a maximum temperature measured by sensors located around a surgical wound and the infrared thermometer measurement. Differences were divided into three intervals.

First Interval contains differences from $-0.4{ }^{\circ} \mathrm{C}$ to $+0.4{ }^{\circ} \mathrm{C}$. Second interval covers differences lower than $-0.4^{\circ} \mathrm{C}$ and third interval differences higher than $+0.4{ }^{\circ} \mathrm{C}$. The percentage of first, second and third interval within evaluated data is $34 \%, 22 \%$ and $43 \%$. These percentages compared to percentages of same intervals of temperature differences measured in abdominal area shows, that temperatures measured around the wound are higher.

Next data analysis evaluates maximum daily temperature measured by all four sensors (three sensors around the wound and a sensor in abdominal area). The temperature around the wound was in all cases higher than the temperature measured in abdominal area. Therefore we will further consider only temperatures around the wound. The data for a group of 24 patients has been examined. A typical length of measurement was 8 days. Patients were divided into three groups. The first group contains patients whose temperature around the wound does not exceed $38^{\circ} \mathrm{C}$. The second group contains patients whose temperature exceeded $38^{\circ} \mathrm{C}$ in less than half of days of measurement. The third group contains patients, whose temperature exceeded $38^{\circ} \mathrm{C}$ in half or more days of measurement.

Fig. 6 shows sample data for patient belonging to second group, temperatures exceeding $38^{\circ} \mathrm{C}$ are highlighted by red color. The percentage of groups within evaluated data is $29 \%, 54 \%$ and $17 \%$. Third group counts 4 patients, whose temperature around the wound exceeded $38{ }^{\circ} \mathrm{C}$ in half or more days of monitoring. 


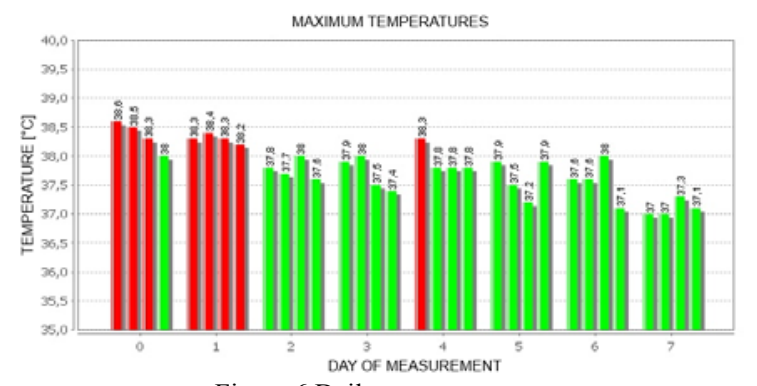

Figure 6 Daily temperatures

\section{SOCIOLOGICAL SURVEY}

A sociological survey examining the views of patients on the continuous monitoring using sensors was carried out together with measurements. The survey involved 22 patients whose ages ranged between 41 and 77 years. Respondents answered several questions focused on the use of sensors.

First question was "Was the use of sensors convenient or inconvenient". Responses are shown in Fig. 7.

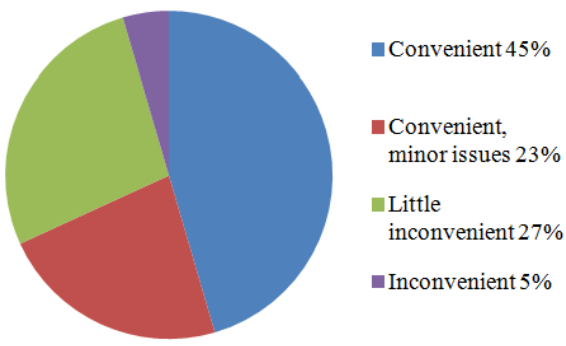

Figure 7 The use of sensors

Next question was "Are you satisfied with the method of fixation of the sensor". Responses are shown in Fig. 8.

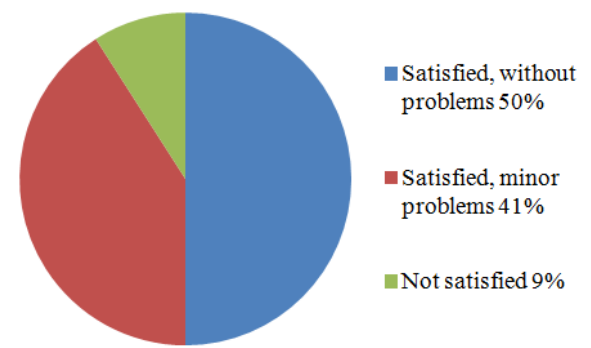

Figure 8 Satisfaction with the method of sensor fixation

Next question was "How do you assess all-day monitoring?" Responses are shown in Fig. 9.

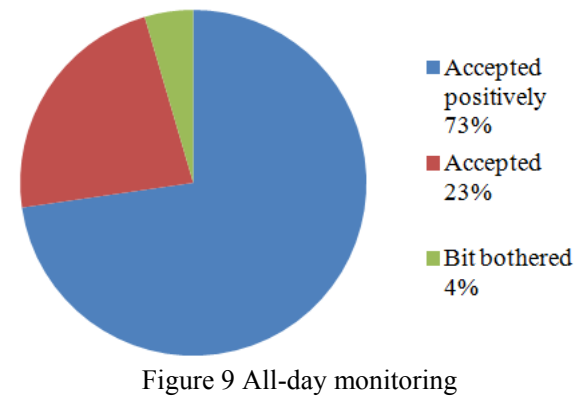

And last question was "Do you agree with monitoring lasting for 2-3 weeks?". Responses are summarized in Fig. 10.

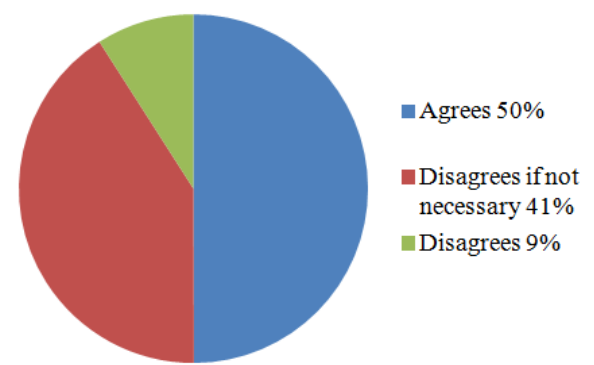

Figure 10 Agreement with 2-3 weeks long monitoring

The survey shown, that the method of monitoring is positively accepted by most of the patients.

\section{CONCLUSION}

The described measurement lasted for several months and provided an opportunity to improve the functionality and reliability of the system. The reliability of the system was successfully verified in the hospital environment. Main issues related to communication range of sensor located on a human body were successfully solved, providing tens of meters communication range from the sensor to the router and allowing coverage of one hospital room with one router. The testing in hospital environment has revealed some issues related to sensor construction and it's fixation on the skin. Issues related to fixation were mainly in loosing proper contact with the skin, which caused faulty temperature measurement. This is now addressed by using a special double sided medical tape, which has a very good adhesion and provides a stable contact with the skin. The sociological survey revealed that the use of tested sensors is accepted positively by most of respondents. If the sensor can be used for monitoring of patient's body temperature, then it can bring several benefits to both patient and hospital. Benefits can be medical stuff time saving, and increased patient's comfort by eliminating the need for manual temperature measurement during night hours, usually leading to patient's awakening.

\section{ACKNOWLEDGMENT}

This research has been supported by the internal project SGS-2015-002 SGS-2016-059 and Regional Innovation Centre for Electrical Engineering (RICE), project No. CZ.1.05/2.1.00/03.0094.

\section{REFERENCES}

[1] Štork M, Tolar D, "Wireless electronic systems for physiological parameters measuring", International Conference on Applied Electronics, Plzeň, Czech Republic, pp 281-284, 2014.

[2] Tolar D, Štork M, " Acknowledged data transmissions in a wireless sensor network with optimized sensor energy consumption", International Conference on Applied Electronics, Plzeň, Czech Republic, pp 263-266, 2015.

[3] IEEE Std 802.15.4-2006: Wireless Medium Access Control (MAC) and Physical Layer (PHY) Specifications for LowRate Wireless Personal Area Networks (LR-WPANs), 2006. 